ON SPECIAL ALGEBRAIC K3 SURFACES. I

This article has been downloaded from IOPscience. Please scroll down to see the full text article.

1973 Math. USSR Izv. 7833

(http://iopscience.iop.org/0025-5726/7/4/A05)

View the table of contents for this issue, or go to the journal homepage for more

Download details:

IP Address: 141.213.236.110

The article was downloaded on 02/07/2013 at 19:51

Please note that terms and conditions apply. 
Izv. Akad. Nauk SSSR

Ser. Mat. Tom 37 (1973), No. 4
Math. USSR Izvestija

Vol. 7 (1973), No. 4

\title{
ON SPECIAL ALGEBRAIC K3 SURFACES. I
}

UDC 513.6

\author{
I. V. DOLGAČEV
}

To I. R. Šafarevič on his fiftieth birthday

\begin{abstract}
In this paper we describe algebraic $\mathrm{K} 3$ surfaces on which lie hyperelliptic curves. We prove a direct and an inverse theorem on the representation of such surfaces as a double plane. We explain the connection between surfaces of this type and elliptic surfaces.
\end{abstract}

\section{Introduction}

Let $k$ be an algebraically closed field of characteristic $p \neq 2$. A smooth projective algebraic surface $X$ over $k$ is called a $\mathrm{K} 3$ surface if $X$ is regular and the canonical class of $X$ is trivial, i.e. if $H^{1}\left(X, \Theta_{X}\right)=0$ and $\omega_{X} \simeq \mathcal{O}_{X}$. We shall call a $\mathrm{K} 3$ surface special if there exists a hyperelliptic curve $C$ of genus $g \geq 2$ on it. The goal of this paper is to give an explicit description of all such surfaces. We will show that the special $\mathrm{K} 3$ surfaces are precisely those which admit a representation as a double plane (i.e. are birationally isomorphic to an affine surface in $A_{k}^{3}$ with the equation $\left.z^{2}=F(x, y)\right)$. Let $\pi$ denote the class of a K3 surface $X$, i.e. the smallest of the dimensions of complete linear systems on $X$ which are greater than one. We shall show that for special $\mathrm{K} 3$ surfaces $\pi$ can only take the values $2,3,4$, or 5 . This answers the question of [2]. Moreover, for $\pi>2$ the condition of speciality is equivalent to the existence of a pencil of elliptic curves of index $\leq 2$ on $X$. In $\$ 4$ we will show that the universal cover of an elliptic Enriques surface (or any Enriques surface if $\operatorname{char}(k)=0$ ) is a special $\mathrm{K} 3$ surface.

The main result is the:

Enriques-Campedelli Theorem. A double plane which is birationally isomorphic to a K3 surface is equivalent to one of the following double planes:

a) $z^{2}=F_{6}(x, y)$, where $F_{6}(x, y)=0$ is a curve of degree 6 ;

b) $z^{2}=F_{8}(x, y)$, where $F_{8}(x, y)=0$ is a curve of degree 8 having two ordinary (singular) quadruple points (these can be infinitely near);

c) $z^{2}=F_{10}(x, y)$, where $F_{10}(x, y)=0$ is a curve of degree 10 having a singular 
point of multiplicity seven and two ordinary triple points which are infinitely near to first order;

d) $z^{2}=F_{12}(x, y)$ where $F_{12}(x, y)=0$ is a curve of degree 12 having a singular point of multiplicity nine and three ordinary points of multiplicity three which are infinitely near to first order.

This theorem was first proved by Enriques in 1896 [8] and later was reproved by Campedelli [6], [7]. In this paper we give a modernization of Campedelli's proof.

The continuation of this paper will be devoted to moduli and automorphisms of special $K 3$ surfaces.

\section{\$1. Basic definitions and auxiliary lemmas}

Definition 1.1. A K3 surface is a smooth projective algebraic surface $X$ with $H^{1}\left(X, \mathcal{O}_{X}\right)=0$ and $\omega_{X}=\Omega_{X}^{2} \simeq \mathcal{O}_{X}$. A K 3 surface is said to be special if there exists a smooth hyperelliptic curve of genus $g \geq 2$ on it.

Lemma 1.2. Let $D=\Sigma_{i} n_{i} D_{i}$ be a connected effective divisor on a $\mathrm{K} 3$ surface $X$. Assume that $n_{i}=1$ for at least one value of $i$. Then

$$
\begin{gathered}
\operatorname{dim}|D| \stackrel{\text { def }}{=} \operatorname{dim}_{k} H^{0}\left(X, O_{X}(D)\right)-1=\frac{\left(D^{2}\right)}{2}+1, \\
p_{a}(D) \stackrel{\text { def }}{=} \operatorname{dim}_{k} H^{1}\left(D, O_{D}\right)=\operatorname{dim}|D| .
\end{gathered}
$$

The exact sequence of sheaves

$$
0 \rightarrow O_{X}(-D) \rightarrow \mathcal{O}_{X} \rightarrow \mathcal{O}_{D} \rightarrow 0
$$

gives an exact sequence of cohomology groups

$$
\begin{gathered}
0 \rightarrow H^{0}\left(X, \mathcal{O}_{X}\right) \rightarrow H^{0}\left(D, \mathcal{O}_{D}\right) \rightarrow H^{1}\left(X, \mathcal{O}_{X}(-D)\right) \\
\rightarrow H^{1}\left(X, \mathcal{O}_{X}\right) \rightarrow H^{1}\left(D, \mathcal{O}_{D}\right) \rightarrow H^{2}\left(X, \mathcal{O}_{X}(-D)\right) \rightarrow H^{2}\left(X, \mathcal{O}_{X}\right) \rightarrow 0 .
\end{gathered}
$$

Since $X$ is a $\mathrm{K} 3$ surface,

$$
H^{1}\left(X, O_{X}\right)=0, \quad H^{2}\left(X, O_{X}\right) \simeq H^{0}\left(X, \omega_{X}\right) \simeq k
$$

On the other hand, because of the conditions on $D$ we have $H^{0}\left(D, \mathcal{O}_{D}\right)=k$. Hence

$$
H^{1}\left(X, \mathcal{O}_{X}(-D)\right)=0, \quad \operatorname{dim}_{k} H^{1}\left(D, \mathcal{O}_{D}\right)=\operatorname{dim}_{k} H^{2}\left(X, O_{X}(-D)\right)-1 .
$$

It remains to use Riemann-Roch for the sheaf $\mathcal{O}_{X}(-D)$ and Serre duality.

Definition 1.3. The number

$$
\pi(X)=\min _{D \subset X}\{\operatorname{dim}|D||\operatorname{dim}| D \mid>1\}
$$

is called the class of the K3 surface $X$.

Remarks. 1. In the case $k=\mathrm{C}$ the class $\pi(X)$ can take any value $\geq 2$ (see [1], Chapter IX). This is apparently also true in general (see [9], p. 256).

2. In [1] K3 surfaces are called Kummer surfaces. At the present time this term is used for a special case of K3's: nonsingular minimal models of the quotient of a 
two-dimensional abelian variety by the involution $x \rightarrow-x$. Arbitrary $\mathrm{K} 3$ surfaces are frequently called generalized Kummer surfaces (cf. [9]). In [2] a "special Kummer surface" was a K3 surface for which the class $\pi$ is realized by a system of hyperelliptic curves. Our definition is obviously somewhat broader. We do not know an example showing that these definitions are actually different.

Definition 1.4. A morphism $f: X^{\prime} \rightarrow X$ of complete integral algebraic surfaces is called a two-sheeted cover (or double cover) if one of the following equivalent conditions holds:

1) / induces a separable quadratic extension of function fields $k\left(X^{\prime}\right) / k(X)$.

2) There exists an open subset $U \subset X^{\prime}$ such that $f \mid U$ is a finite morphism of degree 2.

3) The morphism $/$ splits into a composition $X^{\prime} \stackrel{f_{1}}{\rightarrow} X_{1}^{\prime} \stackrel{f_{2}}{\rightarrow} X$, where $f_{1}$ is a birational morphism and $f_{2}$ is a finite morphism of degree 2 .

A proof of the equivalence of assertions 1)-3) uses a standard technique of the theory of schemes ([10], Chapter 3) and presents no difficulty.

Definition 1.5. A double plane is an algebraic surface which is birationally isomorphic to the affine surface $\operatorname{Spec}\left(k[x, y, z] /\left(z^{2}-F(x, y)\right)\right)$.

Proposition 1.6. An algebraic surface $X$ is a double plane if and only if it is birationally equivalent to a surface $X$ which is a double cover of the projective plane $\mathbf{P}_{k}^{2}$.

The proof is obvious.

Lemma 1.7. Let $g: X^{\prime} \rightarrow X$ be a double cover of smooth surfaces. Then for any divisors $D_{1}$ and $D_{2}$ on $X$ we have

$$
\left(f^{*}\left(D_{1}\right) \cdot f^{*}\left(D_{2}\right)\right)_{X^{\prime}}=2\left(D_{1} \cdot D_{2}\right)_{X}
$$

The proof follows trivially from general properties of Chow rings.

Lemma 1.8. If $D$ is an integral divisor on a $\mathrm{K} 3$ surface, then the linear system $|D|$ has no base points.

For a proof see [1], Chapter VIII, Lemma 2.

Proposition 1.9. Let $C$ be a hyperelliptic curve on a K3 surface $X$ with $p_{a}(C)=$ m. The linear system $|C|$ gives a double cover $f: X \rightarrow V$, where $V$ is a surface of degree $m-1$ in $\mathbf{P}_{k}^{m}$.

For a proof see [1], Chapter VIII, Lemma 3.

Proposition 1.10. Let $f: X^{\prime} \rightarrow X$ be a double cover of smooth surfaces. Let $X^{\prime}$ $\stackrel{f_{1}}{\rightarrow} X_{1}^{\prime} \stackrel{12}{\rightarrow} X$ be the Stein factorization of / (see condition 3) of Definition 1.4). Then the singular points of the surface $X_{1}^{\prime}$ are precisely the inverse images of the singular points of the branch curve $W$ of the finite cover $f_{2}$.

In fact, the surface $X_{1}^{\prime}$ is normal and we can therefore use local properties of finite Galois coverings of algebraic varieties (see [12]). 
Definition 1.11. The curve $W$ of the hypothesis of Proposition 1.10 is called the branch curve of the double cover.

Corollary 1.12. Assume that the branch curve of the double cover $f: X^{\prime} \rightarrow X$ is nonsingular and that the surface $X^{\prime}$ is a minimal model. Then $/$ is a finite morphism.

Lemma 1.13. Let $f: X^{\prime} \rightarrow X$ be a finite morphism of degree two of a normal surface $X^{\prime}$ onto a smooth surface $X$. Assume that the branch curve $W$ of the morphism $f$ has only ordinary double points $P_{1}, \cdots, P_{n}$. Let $\bar{X} \rightarrow X$ be the blowing-up of these points. Then the normalization $\tilde{X}^{\prime}$ of the surface $X^{\prime}{ }^{\prime}{ }_{X} \bar{X}$ is a smooth surface, and the projection $\tilde{X}^{\prime} \rightarrow \bar{X}$ induces a finite cover whose branch curve is the proper trans. form of the curve $W$.

This assertion is a special case of "Jung's method" for resolving the singularities of surfaces. Its verifications reduces to a trivial local computation, which we omit.

\section{§2. The Enriques-Campedelli Theorem}

For the duration of this section $F_{n}$ will denote a relative minimal model of a rational surface possessing a rational curve $S_{n}$ with $\left(S_{n}^{2}\right)=-n$ ( $n$ is a nonnegative integer different from 1) (see [1], Chapter 4). $L$ will denote any one of the fibers of the canonical morphism $F_{n} \rightarrow P_{k}^{1}$ a section of which is the curve $S_{n}$.

Proposition 2.1. Let $\phi: X \rightarrow \mathbf{P}_{k}^{m}$ be a morphism of a normal projective surface whose image $\phi(X)$ is a normally imbedded surface of degree $m-1$. Then there exists a factorization

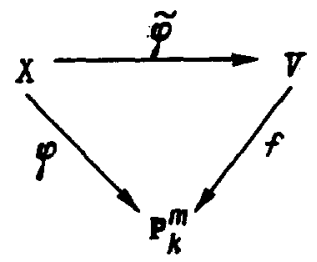

where $f$ is a birational morphism, and $V$ is one of the following surfaces: $\mathbf{P}_{k}^{2}, F_{n}$ or the surface $\bar{F}_{n}$ obtained by blowing down the curve $S_{n}$ on $F_{n}$ at the normal point.

A hyperplane section of the surface $\phi(X)$ is a curve of degree $m-1$ which is normally imbedded in $(m-1)$-dimensional projective space. It follows from the Riemann-Roch Theorem that such a curve is rational. By Proposition 2 of [4] it follows from this that the normalization $V$ of the surface $\phi(X)$ is one of the surfaces enumerated above. Since $X$ is normal, we obtain the desired assertion.

Lemma 2.2. Let $A$ be a regular geometric two-dimensional local ring with residue field $k$ of characteristic different from 2. Let $g$ be an automorphism of $A$ of order 2. Then either the ring of invariants $A^{B}$ is regular, or its completion $\hat{A}^{B}$ is isomorphic to the completion of the local ring of the vertex of a quadratic cone. 
We can obviously assume the ring $A$ to be complete, and hence to be isomorphic to the ring $k[[x, y]]$. In this case the action of $g$ is equivalent to a linear map, and therefore we may assume that either $g(x)=x$ and $g(y)=-y$, or $g(x)=-x$ and $g(y)=$ $-y$. In the former case $A^{8} \simeq k\left[\left[x, y^{2}\right]\right]$ is regular, and in the second $A^{8} \simeq k\left[\left[x^{2}, y^{2}, x y\right]\right]$ $\simeq k[[u, v, w]] /\left(u v-w^{2}\right)$. This proves the lemma.

Theorem 2.3. Let $X$ be a special $\mathrm{K} 3$ surface. Then there exists a double cover $f: X \rightarrow V$, where $V$ is one of the following surfaces: $\mathbf{P}_{k}^{2}, F_{0}, F_{n}(n=2,3,4)$, or $\bar{F}_{2}$.

Let $C$ be a hyperelliptic curve on $X$ and $m$ its genus. Applying Propositions 1.9 and 2.1 , we obtain a double cover $f: X \rightarrow V$, where $V$ is $\mathbf{P}_{k}^{2}, F_{n}$ or $\bar{F}_{n}$. We shall show that the case $n>4$ is impossible, and for $n=3$ and $n=4$ the morphism $f: X \rightarrow \bar{F}_{n}$ passes through the canonical morphism $F_{n} \rightarrow \bar{F}_{n}$, which blows down the section $S_{n}$ at the singular point.

Assume that the first case holds, i.e. $V=F_{n}$ with $n>4$. Let $f: X \stackrel{\phi_{1}}{\longrightarrow} X \stackrel{\phi_{2}}{\longrightarrow} F_{n}$ be the Stein factorization of the morphism / (i.e. $\phi_{1}$ is birational and $\phi_{2}$ is a finite morphism of degree 2). We assume that the morphism $\phi_{1}$ is an isomorphism in some neighborhood of $\phi_{2}^{-1}\left(S_{n}\right)$. Then either $f^{-1}\left(S_{n}\right)$ is $R_{1}+R_{2}$, where the $R_{i}$ are rational curves on $X$, or $f^{-1}\left(S_{n}\right)$ is an irreducible curve. In any case $\left(f^{-1}\left(S_{n}\right)^{2}\right) \geq-8$. Since, on the other hand, $\left(f^{-1}\left(s_{n}\right)^{2}\right)=-2 n$, we obtain from this that $n \leq 4$.

Now suppose $f^{-1}\left(S_{n}\right)=D_{1}+\cdots+D_{r}+R$, where $D_{i}(1 \leq i \leq r)$ are rational curves (perhaps reducible) blown down by the morphism $\phi_{1}$ into distinct singular points of $X^{\prime}$ lying on $\phi_{2}^{-1}\left(S_{n}\right)$, and $R$ is the proper transform of $\phi_{2}^{-1}\left(s_{n}\right)$. We have

$$
\left(f^{-1}\left(S_{n}\right)^{2}\right)=-2 n=\sum_{i=1}^{r}\left(D_{i}^{2}\right)+2 \sum_{i=1}^{r}\left(D_{i} R\right)+R^{2} .
$$

Again it is obvious that either $R$ is an irreducible curve, or $R=R_{1}+R_{2}$, where the $R_{i}$ are rational curves. In either case $\left(R^{2}\right) \geq-8$. Since $\left(D_{i}^{2}\right)=-2$ (cf. Lemma 1.2) and $\left(D_{i} R\right) \geq 1$, we obtain

$$
n=r-\sum_{i=1}^{r}\left(D_{i} R\right)-\frac{\left(R^{2}\right)}{2} \leqslant 4 .
$$

We note that $n>0$ can happen here only in the case $r=1, n=3, R_{1}=R_{2}$.

Now we assume that the second case holds: $V=\vec{F}_{n}(n>2)$. Let $P$ be a singular point of $\bar{F}_{n}$. If the morphism $f: X \rightarrow \bar{F}_{n}$ were finite over a neighborhood of $P$, the local ring $\Theta_{p}$ would be the quotient of a regular sing by the action of the group of order two. By Lemma 2.2 it would follow from this that $P$ is either a nonsingular point or an ordinary double point. The latter obviously contradicts the fact that $n>2$. Thus $f^{-1}(P)$ is a divisor on $X$. By the universality of blowing-up, we will obtain from this that $/$ splits into a composition $X \rightarrow F_{n} \rightarrow \bar{F}_{n}$. This proves the theorem.

Proposition 2.4. Let $f: X \rightarrow F_{0}$ be a double cover with $X$ a $\mathrm{K} 3$ surface. Then the branch curve $W$ of the morphism $f$ has degree (4.4). Moreover, on $X$ there exist elliptic curves $E_{1}$ and $E_{2}$ with $\left(E_{1} \cdot E_{2}\right)=2$, for which the linear system $\left|E_{1}+E_{2}\right|$ 
is irreducible, and any nonsingular curve $D \in\left|E_{1}+E_{2}\right|$ is a hyperelliptic curve of genus 3.

Let $L_{1}=L$ and $L_{2}=S_{0}$ be the effective generators of $\operatorname{Pic}\left(F_{0}\right)$. Then $\left(f^{-1}\left(L_{i}\right)^{2}\right)=0$, and hence $\left|f^{-1}\left(L_{i}\right)\right|$ is a pencil of elliptic curves. If $E_{i}(i=1,2)$ are nonsingular elliptic curves belonging to this pencil, then obviously $\left(E_{1} \cdot E_{2}\right)=$ $2\left(L_{1} \cdot L_{2}\right)=2$. Since $\left(\left(E_{1}+E_{2}\right)^{2}\right)=4$, we have $\operatorname{dim}\left|E_{1}+E_{2}\right|=3$. It is obvious that the system $\left|E_{1}+E_{2}\right|$ is irreducible. For any nonsingular curve $D \in\left|E_{1}+E_{2}\right|$ the pencil $\left|E_{1}\right|$ cuts out a linear series $g_{2}^{1}$ of dimension 1 of degree 2 on $D$. Hence $D$ is a hyperelliptic curve. Its genus is equal to $\left(D^{2} / 2\right)+1=3$. If $W$ is the branch curve of $f$, then $\left(L_{i} \cdot W\right)=4$, since $f^{-1}\left(L_{i}\right)$ is an elliptic curve. Hence $W \sim 4 L_{1}+4 L_{2}$, i.e. it has degree $(4,4)$.

Proposition 2.5. Let $f: X \rightarrow F_{2}$ be a double cover with $X$ a K3 surface. Then the branch curve $W$ of the morphism $f$ is equivalent to the divisor $8 L+4 S_{2}$. Moreover, on $X$ there exist an elliptic curve $E$ and two nonsingular rational curves $R_{1}$ and $R_{2}$ with $\left(R_{1} \cdot R_{2}\right)=0$ and $\left(R_{1} \cdot E\right)=\left(R_{2} \cdot E\right)=1$. Here the linear system $\left|2 E+R_{1}+R_{2}\right|$ is irreducible and any nonsingular curve $D \in\left|2 E+R_{1}+R_{2}\right|$ is a hyperelliptic curve of genus 3.

Since $\left(L^{2}\right)=0,\left|f^{-1}(L)\right|$ is a pencil of elliptic curves. Let $E \in\left|f^{-1}(L)\right|$ be a nonsingular curve belonging to this pencil. Since $\left(S_{2}^{2}\right)=-2$, we have $\left(/^{-1}\left(S_{2}\right)^{2}\right)=-4$. Arguing as in the proof of Theorem 2.3, we will obtain that $f^{-1}\left(S_{2}\right)=R_{1}+R_{2}$, where the $R_{i}$ are isomorphic to $S_{2}$ and $\left(R_{1} \cdot R_{2}\right)=0$. Since $\left(E\left(R_{1}+R_{2}\right)\right)=2\left(L \cdot S_{2}\right)=2$ and $\left(E R_{i}\right)>0$, we have $\left(E \cdot R_{1}\right)=\left(E \cdot R_{2}\right)=1$. Consider the linear system $\left|2 E+R_{1}+R_{2}\right|$. By Lemma 1.2

$$
\operatorname{dim}\left|2 E+R_{1}+R_{2}\right|=\frac{\left(2 E+R_{1}+R_{2}\right)^{2}}{2}+1=3 .
$$

Since, on the other hand, $\operatorname{dim}\left|2 E+R_{i}\right|=2, i=1,2$, it follows that $\left|2 E+R_{1}+R_{2}\right|$ is an irreducible linear system. For any nonsingular curve $D \in\left|2 E+R_{1}+R_{2}\right|$ the pencil $|E|$ cuts out a linear series of dimension 1 and degree 2 on $D$. Hence $D$ is a hyperelliptic curve. Its genus is equal to $\left(D^{2}\right) / 2+1=3$.

Let $W \sim a L+b S_{2}$ be the branch curve of the morphism $/$. Then $(W \cdot L)=4$, since $\left|f^{-1}(L)\right|$ is a pencil of elliptic curves. On the other hand, $\left(W \cdot\left(2 L+S_{2}\right)\right)=8$, since $\left|f^{-1} \cdot\left(2 L+S_{2}\right)\right|=\left|2 E+R_{1}+R_{2}\right|$ is a linear system of hyperelliptic curves of genus 3. Elementary computations now show that $a=8$ and $b=4$.

Proposition 2.6. Let $f: X \rightarrow F_{3}$ be a double cover with $X$ a $\mathrm{K} 3$ surface. Then the branch curve $W$ of the morphism $f$ is equivalent to the divisor $10 L+4 S_{3}$. Moreover, on $X$ there exist an elliptic curve $E$ and nonsingular rational curves $R_{1}$ and $R_{2}$ with $\left(R_{1} \cdot R_{2}\right)=\left(R_{1} \cdot E\right)=1$ and $\left(R_{2} \cdot E\right)=0$. Here the linear system $\left|3 E+2 R_{1}+R_{2}\right|$ is irreducible and any nonsingular curve $D \in\left|3 E+2 R_{1}+R_{2}\right|$ is a hyperelliptic curve of genus 4. 
Again, as in the preceding propositions, $\left|f^{-1}(L)\right|$ is a pencil of elliptic curves. Let $E$ be one of the curves of this pencil. Since $\left(s_{3}^{2}\right)=-3$, we have $\left(f^{-1}\left(s_{3}\right)^{2}\right)=-6$. Arguing as in the first half of the proof of Theorem 2.3, we have that $f^{-1}\left(S_{3}\right)=2 R_{1}+$ $R_{2}$. Here $R_{2}$ is an exceptional curve on $X$ which is mapped by the morphism $/$ into a point, and $2 R_{1}$ is the proper transform of $S_{3}$. Since $\left(\left(2 R_{1}+R_{2}\right)^{2}\right)=-8-2+4\left(R_{1} \cdot R_{2}\right)$ $=-6$, we have $\left(R_{1} \cdot R_{2}\right)=1$. Moreover, $\left(E \cdot\left(2 R_{1}+R_{2}\right)\right)=2\left(L \cdot S_{3}\right)=2$, and since obviously $\left(R_{2} \cdot E\right)=0$, we have $\left(E \cdot R_{1}\right)=1$. We now consider the linear system $\left|3 E+2 R_{1}+R_{2}\right|$. It is obvious that

$$
\operatorname{dim}\left|3 E+2 R_{1}+R_{2}\right|=\frac{\left(\left(3 E+2 R_{1}+R_{2}\right)^{2}\right)}{2}+1=4 .
$$

On the other hand, $\operatorname{dim}\left|3 E+R_{1}+R_{2}\right|<\operatorname{dim}\left|3 E+2 R_{1}\right|=3$. Hence the system $\left|3 E+2 R_{1}+R_{2}\right|$ is irreducible. For any nonsingular curve $D$ of this system $(E \cdot D)=$ $\left(E \cdot\left(3 E+2 R_{1}+R_{2}\right)\right)=2$, and hence the pencil $|E|$ cuts out a linear series of dimension 1 and degree 2 on $D$. Thus $D$ is a hyperelliptic curve of genus $\left(D^{2}\right) / 2+1=4$.

Let $W \sim a L+b S_{3}$ be the branch curve of the morphism $f$. Then $(W \cdot L)=4$, since $\left|f^{-1}(L)\right|$ is a pencil of elliptic curves. On the other hand, $\left(W \cdot\left(3 L+S_{3}\right)\right)=10$, since $\left|f^{-1}\left(3 L+S_{3}\right)\right|=\left|3 E+2 R_{1}+R_{2}\right|$ is a linear system of hyperelliptic curves of genus 4. Simple computations now show that $a=10$ and $b=4$.

Proposition 2.7. Let $f: X \rightarrow F_{4}$ be a double cover with $X$ a K3 surface. Then the branch curve of the morphism $f$ is equivalent to the divisor $12 L+4 S_{4}$. Moreover, on $X$ there exist an elliptic curve $E$ and a nonsingular rational curve $R$ with $(R \cdot E)$ $=1$. Here the linear system $|4 E+2 R|$ is irreducible and any nonsingular curve of this system is a hyperelliptic curve of genus 5.

The proof of this is completely analogous to the proofs of the preceding two propositions, and we omit it.

Proposition 2.8. Let $f: X \rightarrow \bar{F}_{2}$ be a double cover with $X$ a K3 surface. Then there exists a double cover $f^{\prime}: X \rightarrow V$, where either $V=F_{0}$ or $V=F_{2}$.

Let $P$ be the singular point of the surface $\bar{F}_{2}$. If $f^{-1}(P)$ is a divisor on $X$, then, by the universality of blowings-up, the morphism / splits into a composition $X \rightarrow F_{2}$ $\rightarrow \bar{F}_{2}$, where $F_{2} \rightarrow \bar{F}_{2}$ is a blowing down of the section $S_{2}$ into the singular point. But if the morphism $f$ is finite over some neighborhood $U$ of $P$, then, by Lemma 2.2, the morphism / does not split over $U \backslash P$. Since $\bar{F}_{2}$ is isomorphic to a quadratic cone in $\mathbf{P}_{k}^{3}$, we may assume that $f$ is a morphism of $X$ onto a surface of order two in $\mathbf{P}_{k}^{3}$. The inverse image of the hyperplane section $f(X)$ defines a linear system $|D|$ of curves of genus $\pi=3$. On the other hand, the inverse image of a hyperplane section passing through the vertex of the cone defines a divisor $E_{1}+E_{2}$ belonging to $|D|$. Since $\left(D \cdot E_{i}\right)=2$ (the $E_{i}$ are the inverse images of the generators of the cone), we have $\left(E_{i}^{2}\right)=0$ and $\left(E_{1} \cdot E_{2}\right)=2$. Hence the $E_{i}$ are elliptic curves on $X$. Each pencil $\left|E_{i}\right|$ defines a morphism $f_{i}: X \rightarrow \mathbf{P}_{k}^{1}$. Since $\left(E_{1} \cdot E_{2}\right)=2$, the morphism $f_{1} \times$ $f_{2}: X \rightarrow \mathbf{P}_{k}^{1} \times \mathbf{P}_{k}^{1}=F_{0}$ is the desired double cover. 
Theorem 2.9 (Enriques-Campedelli). A special $\mathrm{K} 3$ surface is birationally equivalent to one of the following four types of double planes:

a) $z^{2}=F_{6}(x, y)$, where $F_{6}(x, y)=0$ is a curve of degree 6;

b) $z^{2}=F_{8}(x, y)$, where $F_{8}(x, y)=0$ is a curve of degree 8 which has two ordinary singular points of multiplicity four (perhaps infinitely near: case $\left.b^{\prime}\right)$ );

c) $z^{2}=F_{10}(x, y)$, where $F_{10}(x, y)=0$ is a curve of degree 10 which has a singular point of multiplicity seven and two ordinary triple points which are infinitely near to first order;

d) $z^{2}=F_{12}(x, y)$, where $F_{12}(x, y)=0$ is a curve of degree 12 which has a singular point of multiplicity nine and three ordinary triple points which are infinitely near to first order.

Proof. By Theorem 2.3 and Proposition 2.8 there exists a double cover $f: X \rightarrow V$, where $V$ is one of the surfaces $\mathbf{P}_{k}^{2}$ or $F_{n}(n=0,2,3,4)$.

Case 1. $V=\mathbf{P}_{k}^{2}$. In this case for any line $L$ on $\mathbf{P}_{k}^{2}$ we have $\left(f^{-1}(L)^{2}\right)=2\left(L^{2}\right)=$ 2. Hence the linear system $\left|/^{-1}(L)\right|$ consists of curves of genus 2 , and the intersection index of $L$ with the branch curve equals 6 . Thus $n(X)=2$, and the branch curve of $f$ has degree 6 . From this it follows that the surface $X$ is birationally equivalent to a double plane of type a).

Case 2. $V=F_{0}$. By Proposition 2.4 the branch curve $W$ of the morphism $f: X \rightarrow V$ has degree $(4,4)$. Let $P$ be a point on $F_{Q}$ which does not belong to $W$, let $\widetilde{F}_{0} \rightarrow F$ be the blow-up of $P, \tilde{X}=X \times{ }_{F_{0}} \widetilde{F}_{0}$, and $f: \widetilde{X} \rightarrow \widetilde{F}_{0}$ the projection. After taking the composition of $\tilde{f}$ with the canonical morphism $\tilde{F}_{0} \rightarrow P_{k}^{2}$ (blowing-down of the proper transforms on $\widetilde{F}_{0}$ of the generators of $F_{0}$ passing through $P$ ), we obtain a double cover $\tilde{X} \rightarrow P_{k}^{2}$. The branch curve of this cover is the proper image of $W$ relative to the birational map $F_{0} \rightarrow \widetilde{F}_{0} \rightarrow \mathbf{P}_{k}^{2}$ and is obviously a curve of degree 8 with two singular points of multiplicity four (the images of curves on $F_{0}$ which can be blown down).

Since the surface $\tilde{X}$ is birationally equivalent to the desired surface $X$, we see that $X$ is birationally equivalent to a double plane of type b).

Case 3. $V=F_{2}$. By Proposition 2.5 the branch curve $W$ of the morphism $f: X \rightarrow V$ is equivalent to the divisor $8 L+4 S_{2}$.

Let $P_{1}$ and $P_{2}$ be distinct closed points on $F_{2}$ and not belonging to $W$. Let $\tilde{F} \rightarrow F_{2}$ be the blow-up of these points. Blowing down the proper images of generators $L_{1}$ and $L_{2}$ passing through $P_{1}$ and $P_{2}$, we obtain a double cover of surfaces $\tilde{X}=$ $X \times{ }_{F_{2}} \widetilde{F}_{2} \rightarrow F_{0}$ whose branch curve $\tilde{W}$ is a curve of degree $(8,4)$ with two ordinary quadruple points lying on the section $S_{0}$. Let $Q$ be one of these points and $L$ a generator passing through $Q$. Blowing up the point $Q$ and blowing down the proper images of the sections $S_{0}$ and $L$, we obtain a double cover $\widetilde{X}=\widetilde{X} \times{ }_{F_{0}} \widetilde{F}_{0} \rightarrow P_{k}^{2}$ with a branch curve of degree eight with two infinitely near singular points of multiplicity four. Since the surfaces $X$ and $X$ are birationally isomorphic, we will obtain that $X$ is birationally isomorphic to a double plane of type $b^{\prime}$ ).

Case 4. $V=F_{3}$. By Proposition 2.6 the branch curve $W$ of the morphism $f: X \rightarrow V$ is equivalent to the divisor $10 L+4 S_{3}$. In this case the curve $W$ contains as one of its 
irreducible components the section $S_{3}$. Let $W^{\prime}=W \backslash S_{3}$. Then $\left(W^{\prime} \cdot S_{3}\right)=1$ and $\left(W^{\prime} \cdot L\right)=3$. Making elementary transformations at any three points $P_{1}, P_{2}, P_{3}$ not lying on $W$, we obtain a double cover $\tilde{f}: \tilde{X} \rightarrow F_{0}$, where $\tilde{X}$ is birationally equivalent to $X$ and the branch curve of $\tilde{f}$ is the section $S_{0}$ and some curve $W^{\prime}$ of degree $(10,3)$ with three singular points of multiplicity four on $S_{0}$. Now making an elementary trans-

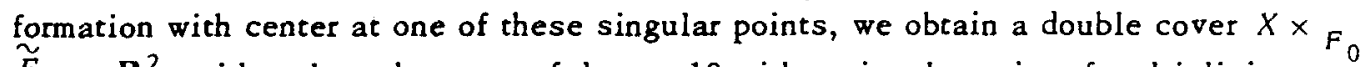
$\widetilde{F}_{0} \rightarrow \mathbf{P}_{k}^{2}$ with a branch curve of degree 10 with a singular point of multiplicity seven and two ordinary triple points which are infinitely near to it. Thus we get case c) of the theorem.

Case 5. $V=F_{4}$. By Proposition 2.7 the branch curve $W$ of the morphism $f: X \rightarrow F_{4}$ is equivalent to the divisor $12 L+4 S_{4}$. In this case the curve $S_{4}$ is contained in $W$, and the curve $W^{\prime}=W-S_{4}$ does not intersect $S_{4}$.

Carrying out a construction analogous to case 4 , we obtain case d) of the theorem.

Conollary 2.10. For any special $\mathrm{K} 3$ surface the class $\pi$ can only take the values $2,3,4$ or 5 .

In fact, the inverse image of a line on a double plane of type a)-d) is a hyperelliptic curve of genus $2,3,4$ or 5 .

Corollary 2.11. Each special K3 surface with class $\pi=2$ (respectively 3, 4, 5) is a double cover of $\mathbf{P}_{k}^{2}$ (respectively $F_{0}$ or $F_{2}, F_{3}, F_{4}$ ).

As was mentioned at the beginning of the proof of Theorem 2.9, each special $\mathrm{K} 3$ surface $X$ is a double cover of one of the surfaces $P_{k}^{2}, F_{0}, F_{2}, F_{3}$ or $F_{4}$. If $\pi=2$, then the linear system $|C|$ of curves of genus 2 defines a double cover $X \rightarrow P_{k}^{2}$. If $\pi=3$, the linear system $|C|$ of curves of genus 3 defines a morphism $\mid: X \rightarrow \mathrm{P}_{k}^{3}$ whose image $V$ is a surface of degree two. Each such surface is either the quadric $F_{0}$ or the cone $\bar{F}_{2}$. Now apply Proposition 2.8. If $\pi=5$, then $X$ can be mapped two-to-one only onto $F_{4}$, since otherwise by Propositions $2.4-2.6$ there would exist a curve of smaller genus on $X$. For $\pi=4$ the morphism $X \rightarrow \mathrm{P}_{k}^{4}$, defined by a system of curves of genus 4, can pass through only one of the surfaces $F_{n}(n=2,3,4)$ (see the proof of Theorem 2.3). The case $n=2$ is impossible by Proposicion 2.5. The case $n=4$ is impossible since there does not exist an embedding of $F_{4}$ into $\mathbf{P}_{k}^{4}$.

§3. Converse of the Enriques-Campedelli Theorem

Lemma 3.1. Let $X$ be a normal algebraic surface and $D$ an integral curve on $X$ whose image in the group $\mathrm{Pic}(X)$ is divisible by two. Then there exists an irreducible unramified cover $F: X^{\prime} \rightarrow X \backslash D$ of degree 2.

Using the étale topology, we consider on $U=X \backslash D$ the Kummer sequence [3]:

$$
0 \rightarrow \Gamma\left(U, O_{U}^{*}\right) / \Gamma\left(U, O_{U}^{*}\right)^{2} \rightarrow H^{1}\left(U, \mu_{2}\right) \rightarrow \operatorname{Pic}\left(U_{2}\right) \rightarrow 0
$$

(recall that char $k \neq 2$ ). Since $X$ is nomal, the canonical restriction morphism $r: \operatorname{Pic}(X) \rightarrow \operatorname{Pic}(U)$ is surjective, and its kernel is generated by the divisor $D$. Since 
the class of $D$ is divisible in $\operatorname{Pic}(X)$ by 2, the quotient group $\operatorname{Pic}(X) / \operatorname{Ker}(r)=\operatorname{Pic}(U)$ contains an element of order two. Hence

$$
H^{1}\left(U, \mu_{2}\right) \neq 0
$$

It remains to use the fact that the group $H^{1}\left(U, \mu_{2}\right)$ classifies the principal bundles over $U$ with structure group $Z / 2 Z$. A nontrivial such bundle also defines the desired unramified cover $f: X^{\prime} \rightarrow U$.

Lemma 3.2. In the notation of Lemma 1.13 we have

$$
c_{2}\left(\widetilde{X}^{\prime}\right)=2\left(c_{2}(X)+n\right)+2 \sum_{i=1}^{h}\left(g\left(W_{i}\right)-1\right),
$$

where $c_{2}(Z)$ is the second Chern number of the tangent sheal to the surface $Z$ (= the topological Euler characteristic of $Z$ in case $k=\mathrm{C}$ and the l-adic Euler characteris. tic in the general case), and $g\left(W_{i}\right), I \leq i \leq h$, is the geometric genus of an irreducible component of the branch curve $W$.

If $\bar{X}$ is the blow-up of the double points of $W$ on $X$, then $c_{2}(\bar{X})=c_{2}(X)+n$. Now use the "Severi correspondence formula" [11].

Theorem 3.3. Let $z^{2}=F_{2 n}(x, y)(n=3,4,5,6)$ be a double plane. Assume that the branch curve $F_{2 n}(x, y)=0$ is irreducible and, except for the singularities mentioned in Theorem 2.9, has only ordinary double points. Then the minimal projective nonsingular model of this double plane is a special $\mathrm{K} 3$ surface with class $\pi \leq n-1$.

Prool. Suppose $n=3$, and $W \subset \mathbf{P}_{k}^{2}$ is a projective curve of degree 6 which is irreducible and has only ordinary double points (or is smooth). By Lemma 3.1 there exists an unramified cover $U^{\prime} \rightarrow U=\mathbf{P}_{k}^{2} \backslash W$ of degree two. This cover can obviously be extended to a finite morphism $f: X \rightarrow \mathbf{P}_{k}^{2}$, ramified only over $W$ (cf. [12], p. 4). Let $V$ be the blow-up of $\mathbf{P}_{k}^{2}$ with center at the singularities of $W$. By Lemma 1.13 the normalization $X^{\prime \prime}$ of the surface $X \times{ }_{P_{k}^{2}} V$ is a nonsingular surface, and the projection $f^{\prime}: \bar{X}^{\prime} \rightarrow V$ defines a finite double cover, ramified over the proper transform $\bar{W}$ of the curve $W$. Let $L_{1}, \cdots, L_{n}$ be exceptional curves on $V$ which are inverse images of singular points of $W$ relative to the projection $V \rightarrow P_{k}^{2}$. We have $K_{V}=-3 \bar{H}+L_{1}+\cdots+L_{n}$, where $\bar{H}$ is the proper transform of the line $H$ in $\mathbf{P}_{k}^{2}$.

On the other hand, it is obvious that $\bar{W} \sim 6 \bar{H}-2 L_{1}+\cdots+2 L_{n}$. From this it follows (cf. [11]) that $K_{X} \sim f^{\prime *}\left(K_{V}\right)+1 / 2 f^{\prime *}(\bar{W}) \sim 0$. By Lemma 3.2, $c_{2}\left(X^{\prime}\right)=2(3+n)+$ $2(9-n)=24$. By Noether's formula

$$
1-q+p_{g}=\frac{c_{2}\left(X^{\prime}\right)+\left(K_{X^{\prime}}^{2}\right)}{12},
$$

from which it follows that $q=\operatorname{dim}_{k} H^{\prime}\left(X, \mathcal{O}_{X}\right)=0$. Hence the surface $X^{\prime}$ is regular and $K_{X} \sim 0$, and this is the definition of a $K 3$ surface.

Now suppose $n=4$ and $W \subset P_{k}^{2}$ is an irreducible projective curve of degree 8 , 
having perhaps, besides the two ordinary quadruple points $A_{1}$ and $A_{2}$, only ordinary double points $P_{1}, \cdots, P_{n}$. Blowing up the points $A_{1}$ and $A_{2}$ on $\mathbf{P}_{k}^{2}$ and blowing down the proper transform of the line $A_{1} A_{2}$, we get that the proper transform $\bar{W}$ of $W$ on the quadric $F_{0}$ is an irreducible curve of type $(4,4)$, having perhaps ordinary double points $P_{1}^{\prime}, \ldots, P_{n}^{\prime}$. The remaining part of the argument is carried out in this case analogously to that of the previous case (replacing $\mathbf{P}_{k}^{2}$ by $F_{0}$ ).

The proofs of the remaining cases follow along the model of the preceding ones, and we omit them. We only note that for $n=4$ (case of infinitely near points) it is necessary to replace $P_{k}^{2}$ by $F_{2}$, for $n=5$, by $F_{3}$, for $n=6$ by $F_{4}$ (cf. Propositions 2.5, 2.6 and 2.7) and to use Lemmas 3.1 and 3.2.

Remark 3.4. In case $k=\mathrm{C}$ it follows easily from the results of G. N. Tjurina (see [1], Chapter IX) that for a "generic" double plane of type a), b) or b'), c), d) (cf. Theorem 2.9) $\pi=2,3,4,5$ respectively. This is apparently also true in general.

\section{\$. Elliptic surfaces and special K3 surfaces}

We recall some definitions.

Definition 4.1. A pencil of elliptic curves on a projective algebraic surface $X$ is a morphism $f: X \rightarrow B$, where $B$ is a smooth curve, and the general fiber of $f$ is a smooth elliptic curve. The index of a pencil is the index of the generic fiber $X_{\eta}$ of the morphism $f$, i.e. the greatest common divisor of the degrees of effective divisors on $X_{\eta}$ defined over the field $k(B)$.

Let $r: \mathrm{Pic}(X) \rightarrow \mathrm{Pic}\left(X_{\eta}\right)$ be the canonical restriction morphism. Passing to Néron-Severi groups we have a homomorphism $\bar{r}: \mathrm{NS}(X) \rightarrow \mathrm{NS}\left(X_{\eta}\right) \rightarrow Z$. It is easy to see that the index of the pencil $f$ on $X$ equals the order of the cokernel of the homomorphism $\vec{r}$. In other words, it is equal to $\min \{(F \cdot C) \mid C$ a transversal curve over $B$, $F$ an arbitrary fiber of $f\}$.

Definition 4.2. A smooth projective algebraic surface is said to be elliptic if there exists a pencil of elliptic curves on it. The index $l$ of an elliptic surface $X$ is the minimal index of all the possible pencils of elliptic curves on $X$.

Lemma 4.3. For an algebraic $\mathrm{K} 3$ surface $X$ to be an elliptic surface with index $I$ it is necessary and sufficient that on $X$ there exist a connected curve $C$ with $p_{a}(C)=1$ and an irreducible curve $S$ with $(C \cdot S)=I$.

Necessity is obvious. To prove the sufficiency we need to use Lemmas 1.2 and 1.8 and consider the morphism $f: X \rightarrow \mathbf{P}_{k \text { - }}^{1}$ defined by the linear system $|C|$.

Theorem 4.4. Every special K3 surface $X$ of class $\pi>2$ is an elliptic surface. Moreover, for $\pi=4$ or 5 the index of this surface is equal to one (1), and for $\pi=3$ it is $\leq 2$.

Prool. By 2.11 for $\pi=3$ (respectively 4 or 5 ) there exists a double cover $X$ over $F_{0}$ or $F_{2}$ (respectively $F_{3}$ or $F_{4}$ ). It remains to apply Lemma 4.3 and Propositions 2.4 and 2.5 (respectively 2.6 or 2.7 ). 
Theorem 4.5. Each elliptic $\mathrm{K} 3$ surface with index $I \leq 2$ is a special $\mathrm{K} 3$ surface.

Proof. Assume $I=1$ and that $C$ and $S$ are curves on $X$ chosen via Lemma 4.3. Since $\left(s^{2}\right) \geq-2$, we have

$$
\left((4 C+2 S)^{2}\right)=16(C \cdot S)+4\left(S^{2}\right)=16+4\left(S^{2}\right)>8
$$

Moreover,

$$
\begin{gathered}
\left((4 C+2 S)^{2}\right)>\left((4 C+S)^{2}\right)=8+\left(S^{2}\right), \\
\left((4 C+2 S)^{2}\right)>\left((3 C+2 S)^{2}\right)=12+4\left(S^{2}\right) .
\end{gathered}
$$

Thus the linear system $|4 C+2 S|$ has no fixed components and consists of curves of genus $\geq 5$. Since $\left(C^{2}\right)=0$, we have $(C \cdot(4 C+2 S))=2$.

Hence the pencil $|C|$ cuts out a linear series $g_{2}^{1}$ on a nonsingular curve $D \in$ $|4 C+2 S|$. Hence $D$ is hyperelliptic and $X$ is special.

Suppose $I=2$. The linear system $|C+S|$ has no fixed components and consists of curves of genus

$$
g=\frac{\left((C+S)^{2}\right)}{2}+1=\frac{4+\left(S^{2}\right)}{2}+1 \geqslant 2
$$

Since $(C(C+S))=(C \cdot S)=2$, the pencil $|C|$ cuts out a linear series $g_{2}^{1}$ on any nonsingular curve $D \in|C+S|$. Hence $D$ is a byperelliptic curve and $X$ is a special $K 3$ surface.

Definition 4.6. An Enriques surface is a smooth algebraic surface $X$ with $H^{1}\left(X, \mathcal{O}_{X}\right)=H^{2}\left(X, \mathcal{O}_{X}\right)=0$ and $\omega_{X}^{\otimes 2} \simeq \mathcal{O}_{X}$.

Proposition 4.7. Let $X$ be an Enriques surface. Then there exists a finite unramified cover of degree two $f: X^{\prime} \rightarrow X$, where $X^{\prime}$ is a $\mathrm{K} 3$ surface.

Since $\omega_{X} \neq \mathcal{O}_{X}$, the sheaf $\omega_{X}$ defines an element of order two in the group Pic $(X)$. By the Kummer sequence

$$
0 \rightarrow H^{0}\left(X, \mathcal{O}_{X}^{*}\right) / H^{0}\left(X, \mathcal{O}_{X}^{*}\right)^{2} \rightarrow H^{1}\left(X, \mu_{2}\right) \rightarrow \operatorname{Pic}\left(X_{1}\right) \rightarrow 0
$$

we will obtain that $H^{1}\left(X, \mu_{2}\right) \neq 0$. Since the group $H^{1}\left(X, \mu_{2}\right)$ classifies principal homogeneous spaces with structure group $Z / 2 Z$, a nontrivial p.h.s. defines a finite unramified double cover $f: X^{\prime} \rightarrow X$. Elementary computations (see [11]) show that $\omega_{X^{\prime}}$ $\simeq f^{*}\left(\omega_{X}\right) \simeq \mathcal{O}_{X^{\prime}}$ and $H^{1}\left(X^{\prime}, \mathcal{O}_{X}{ }^{\prime}\right)=0$. Hence $X^{\prime}$ is a $\mathrm{K} 3$ surface.

Lemma 4.8 (L. Godeaux). Let $X$ be an elliptic surface with $H^{1}\left(X, \mathcal{O}_{X}\right)=$ $H^{2}\left(X, \Theta_{X}\right)=0$, let $f: X \rightarrow \mathbf{P}_{k}^{1}$ be the corresponding pencil of elliptic curves, and $F$ an arbitrary fiber over a closed point. Let $\Gamma_{1} \sim\left(1 / m_{1}\right) F, \cdots, \Gamma_{n} \sim\left(1 / m_{n}\right) F$ be the "supports" of multiples of fibers of f. Then

$$
\omega_{X} \simeq \mathcal{O}_{X}\left((n-1) F-\Gamma_{1}-\ldots-\Gamma_{n}\right) \text {. }
$$

Let $\omega_{X}=\mathcal{O}_{X}\left(K_{X}\right)$. Since $H^{1}\left(X, \mathcal{O}_{X}\right)=0$, for any complete (integral) divisor $D$ with $p_{a}(D)=1$ we have $\operatorname{dim}|D+K|=0$. Let $D^{\prime}$ denote the uniquely defined 
effective divisor of the linear system $|D+K|$. In particular, $F^{\prime}=\lambda_{1} \Gamma_{1}+\cdots+\lambda_{n} \Gamma_{n}$, where $\lambda_{i}<m_{i}$ (since $\left.H^{2}\left(X, \mathcal{O}_{X}\right)=H^{0}\left(X, \omega_{X}\right)=0\right)$.

On the other hand, since $F^{\prime}=\left(m_{1}-1\right) \Gamma_{1}+\Gamma_{1}^{\prime}$, we have $\Gamma_{1}=\left(\lambda_{1}-m_{1}+1\right) \Gamma_{1}+$ $\cdots+\lambda_{n} \Gamma_{n}$. From this we obtain $\lambda_{1}-m_{1}+1 \geq 0$, which gives $m_{1}-1 \leq \lambda_{1}$, and since $\lambda_{1}<m_{1}$, then $\lambda_{1}=m_{1}-1$. The equalities $\lambda_{i}=m_{i}-1$ are proved analogously.

Finally we obtain

$$
\begin{gathered}
K x \sim F^{\prime}-F \sim\left(m_{1}-1\right) \Gamma_{1}+\ldots+\left(m_{n}-1\right) \Gamma_{n}-F \\
\sim(n-1) F-\Gamma_{1}-\ldots-\Gamma_{n} .
\end{gathered}
$$

Corollary 4.9. In the notation of the preceding lemma, if $\omega_{X}^{\otimes 2} \simeq \mathcal{O}_{X}$, then $n=2$, $m_{1}=m_{2}=2$ and $\omega_{X} \simeq \mathcal{O}_{X}\left(\Gamma_{1}-\Gamma_{2}\right)$.

We have

$$
\begin{aligned}
& O_{X} \simeq \omega_{X}^{\otimes 2} \simeq \mathcal{O}_{X}\left((2 n-2) F-2 \Gamma_{1}-\ldots-2 \Gamma_{n}\right) \\
& \quad=\mathcal{O}_{X}\left((n-2) F+\left(m_{1}-2\right) \Gamma_{1}+\ldots+\left(m_{n}-2\right) \Gamma_{n}\right)
\end{aligned}
$$

Since all the $m_{i} \geq 2$, we have $n=2$ and $m_{i}=2$. Moreover,

$$
\omega_{X} \simeq \mathcal{O}_{X}\left(F-\Gamma_{1}-\Gamma_{2}\right) \simeq \mathcal{O}_{X}\left(\Gamma_{1}-\Gamma_{2}\right)
$$

Theorem 4.10. Let $X$ be an elliptic Enriques surface. Then there exists a finite unramified double cover $f: X^{\prime} \rightarrow X$, where $X^{\prime}$ is a special $\mathrm{K} 3$ surface.

Proof. Let / be the cover constructed in Lemma 4.1. We shall show that $X^{\prime}$ is a special K3 surface. Let $J$ be the Jacobian surface of $F$ ([1], Chapter VI). It is easy to see that $J$ is a rational surface (cf. [s]). Hence the Safarevič group $11\left(J_{\eta}\right)$ of the generic fiber of $J$ is trivial. Using the theory of Ogg and Šafarevič, we obtain that the group of principal homogeneous spaces $W C\left(J_{\eta}\right)$ is the direct sum of the groups of "local invariants" $W C\left(J_{\eta}\right)=\oplus_{x \in \mathrm{P}_{k}^{1}(k)} H^{1}\left(J_{x}, \mathbf{Q} / \mathbf{Z}\right)$.

By Corollary 4.9 the surface $X$ defines an element $X_{\eta}$ in $w C\left(J_{\eta}\right)$ of the form $(\alpha, \beta)$, where $2 \alpha=2 \beta=0$. Hence the order of $X_{\eta}$ in this group equals two. Since the index of $X_{\eta}$ is equal to its order [13], $X_{\eta}$ has a rational point over the quadratic extension $k(\eta)$. The composition $X^{\prime} \rightarrow X \rightarrow \mathbf{P}_{k}^{1}$ defines a structure of elliptic surface on $X^{\prime}$. Since the index of $X_{\eta}^{\prime}$ obviously does not exceed the index of $X_{\eta}$, we then conclude that $X^{\prime}$ is an elliptic $\mathrm{K} 3$ surface of index $\leq 2$. It remains to apply Theorem 4.5.

Remarks. 1) In case char $(k)=0$ one can show that every Enriques surface is an elliptic surface (see [1], Chapter X). This-apparently is also true in general.

2) One can show that for an elliptic Enriques surface $X$ the torsion subgroup Tors $(\mathrm{Pic}(F)) \simeq \mathrm{Z} / 2 \mathrm{Z}$. Moreover, since $H^{1}\left(X, O_{X}\right)=0$, it follows from Kummer and Artin-Schreier theory that the surface $X^{\prime}$ constructed in Theorem 4.4 is the unique finite abelian unramified cover of $X$. In characteristic zero this fact follows trivially from the simply-connectedness of $\mathrm{K} 3$ surfaces.

3) An elliptic Enriques surface $X$ is said to be special (cf. [1], Chapter $X$ ) if 
there exists a quasi-section of degree 2 which is a rational curve. In this case the double plane corresponding to the $\mathrm{K} 3$ surface $X^{\prime}$ can be constructed explicitly. It has the form $z^{2}=F_{3}(x, y) \cdot F_{3}^{\prime}(x, y)$, where $F_{3}(x, y)=0$ and $F_{3}^{\prime}(x, y)=0$ are cubic curves. In the general case $\pi \leq 3$ for the surface $X^{\prime}$.

\section{Received 14/SEPT/72}

\section{BIBLIOGRAPHY}

1. R. Šafarevič et al., Algebraic surfaces, Trudy Mat. Inst. Steklov, $75(1965)=$ Proc. Steklov Inst. Math.75 (1965). MR 32 \#7557; 35 \#6685.

2. B. G. Averbuh, On special types of Kummer and Enriques surfaces, Izv. Akad. Nauk SSSR Ser. Mat. 29 (1965), 1095-1118; English transl., Appendix to Proc. Steklov Inst. Math. 75 (1965), 251-276. MR $35 \# 6686$.

3. M. Artin, Covering cohomologies of schemes, Uspehi Mat. Nauk 20 (1965), no. 6 (126), 13-18. (Russian) MR $34 \$ 7530$.

4. M. H. Gizatullin, On affine surfaces that can be completed by a nonsingular rational curve, Izv. Akad. Nauk SSSR Ser. Mat. 34 (1970), 778-802 = Math. USSR Izv. 4 (1970), 787-810. MR $43 \# 6211$.

5. 1. V. Dolgačev, On Severi's conjecture conceming simply connected algebraic surfaces, Dokl. Akad. Nauk SSSR 170 (1966), 249-252 = Soviec Math. Dokl, 7 (1966), 1169-1172. MR 34 \#2579.

6. L. Campedelli, Sopra i piani doppi con tutti $i$ generi uguali all'unita, Rend. Sem. Mat. Univ. Padova 11 (1940), 1-27. MR 8, 224.

7. - Le superficie con $i$ generi uguali all'unita rappresentabili in infiniti modi sopra un piano doppio, Univ. Roma e Ist. Naz. Alta Mat. Rend. Mat. e Appl. (5) 1 (1940), 105-138. MR 9, 57.

8. F. Enriques, Sui piani doppi di generi uno, Mem. Soc. Ital. Sci. (3) 10 (1896), 201-222.

9. —_, Le superficie algebriche, Zanichelli, Bologna, 1949. MR 11, 202.

10. A. Grothendieck, Éléments de géométrie algébrique, Inst. Hautes Études Sci. Publ. Math. No. 17 (1963). MR $29 \# 1210$.

Translated by J. S. JOEL 